\title{
Design and Implementation of Robust Hybrid Control of Vision Based Underactuated Mechanical Nonminimum Phase Systems
}

\author{
Haoping Wang ${ }^{1}$, Christian Vasseur ${ }^{2}$, Vladan Koncar ${ }^{3}$, Afzal Chamroo ${ }^{4}$, Nicolai Christov ${ }^{5}$ \\ ${ }^{1,2,5}$ LAGIS CNRS 8146, \\ Université des Sciences et Technologies de Lille, \\ UFR IEEA, Bâtiment P2, \\ 59655 Villeneuve d'Ascq Cedex, France, \\ haoping.wang, christian.vasseur, nicolai.christov@univ-lille1.fr \\ ${ }^{3}$ GEMTEX, ENSAIT, \\ 9 Rue de l'Ermitage, \\ BP 30329, 59056 Roubaix, France, \\ vladan.koncar@ensait.fr \\ ${ }^{4}$ LAII, Université Poitiers, \\ 40 Avenue du Recteur Pineau, \\ 86022 Poitiers, France
}

\begin{abstract}
This paper presents a vision based Cart-Inverted Pendulum (CIP) system under a hybrid feedback configuration: the continuous cart's position measured by encoder and the delayed \& sampled inverted pendulum's upper coordinates, obtained from a visual sensor. The challenge here is to stabilize the CIP from a big inclined initial angle by using a low cost CCD camera. Under this scheme, we propose a hybrid control which consists in a Jumpingup (Bang-Bang) control and a two causal stabilization loops control: the first one (inner loop) realizes a linearization and the stabilization control of the pendulum based on an innovative Piecewise Continuous Reduced Order Luenberger Observer coupled with a linearization module, the second one (the outer loop) realizes a Lyapunov based control for the unstable internal system with lower dynamics than that of the pendulum. This hybrid control method is capable of balancing the CIP system within small cart' $\mathrm{s}$ displacement. Performances issues of the proposed method are illustrated by the experimental figures and videos.
\end{abstract}

Keywords: Visual servoing, underacturated mechanical systems, nonminimum phase systems, Lyapunov functions, piecewise continuous systems.

\section{Introduction}

During the last few years, there has been a considerable amount of interest in the control of vision based underactuated mechanical systems forced by fewer actuators than degrees of freedom, presents a challenging problem. The interest comes from the need of supervision in remote control especially via Internet based network, more flexible contactless wiring and improved signal/noise ratio. Various models of vision based underactuated mechanical control have been reported in attempt to improve the visual servoing's performance.

Recalling [1] the visual servoing term is defined as using visual feedback to control a robot. For example visual (image based) features such as points, lines and regions can be used to enable the alignment of a manipulator/gripping mechanism with an object. Vision is a part of a control system providing feedback. However, traditionally visual sensing and manipulation are combined in an open-loop configuration, 'looking' and 'moving', or just for visualizations and animations purposes referring to pendulum control in remote control laboratory [2], [3]. Recently, visual supervision has been gradually combined in the closed control loop particularly for cart-inverted pendulum control such as in [4], [5]. Unfortunately there is no real successful application reported on controlling the cart's position and pendulum' $\mathrm{s}$ angle by visual servoing till now. A fuzzy-logic based controller was reported in [6], but only for controlling a rotary pendulum near the unstable equilibrium zone not exceeding $\pm 5^{\circ}$. For larger deviations, the system turned out to be too slow to compensate. This control was limited in time on a few seconds in keeping the pendulum upright. In [7] a just-in-time human simulated method was developed to stabilize a two-link Direct Drive Arm-pendulum system. The direction of the pendulum movement is restricted on tangential plane for the trajectory of the tip of second link. This human learning 
and memory based fuzzy control can stabilize the inverted pendulum only for seconds $(\leq 16$ s) and with larger angular position oscillations $\pm 26^{\circ}$, just like humans.

Analyzing the difficulties of previous vision based research works related to CIP control; it seemed that the camera signal has not been sufficiently exploited. The problem is that these sensors often deliver sampled and delayed signals due to their digital nature and computation-transfer time (image processing) respectively. Our challenge here is to consider the low cost CCD cameras as contact-less pendulum sensor to stabilize the CIP jumping from a big angular position with a big time delay.

Our efforts have been focused on the development of an accurate observer using the theory of Piecewise Continuous Systems (PCS) [8]. This kind of systems are continuous controlled hybrid systems with independent switching and controlled input [8], [9]. Considering the sampled delayed camera's measurements (pendulum's angular position) as autonomous switching and controlled impulse, we estimate the present continuous pendulum's angular position and angular speed. With the improved pendulum's angular position and the estimated angular speed, we can construct our control methods. The research work presented here is an extension and development of the preceding works [8], [9], [10], [11].

The paper is organized as follows: sections 2 , 3 and 4 present the description and modelling of the real vision based CIP system, particularly the TSAI calibration method and the way to calculate the delayed and sampled pendulum' $s$ angular position. In section 5 a hybrid control consisting of the Jumping-up control and the two causal stabilization loops under a logic-based switch mechanism is proposed. A Piecewise Continuous Reduced Order Luenberger Observer (PCROLO) is also developed. Simulation and experimental results are given in section 6 .

\section{Experimental Setup}

The vision based cart-inverted pendulum experimental system illustrated in Figure 1, contains the following four parts:

1) The mechanical system: The system is composed of an aluminium chassis, enabling only $48 \mathrm{~cm}$ displacement and a plastic inverted pendulum mounted on the mobile cart. Between the pendulum and the cart, an installed shock absorber prevents the pendulum from completely falling to horizontal position. It allows the pendulum to have a maximum angle $50^{\circ}$ with respect to the vertical position.

2) The controller: The controller is implemented on the dSpace based Digital Signal Processing card DS1103 via ControlDesk integrated with Matlab/ Simulink. We can model, supervise and develop directly control methods for the real-time system by the benefits of the access to the control card's variables. The control signals are sent to a power amplifier via $\pm 10 \mathrm{~V}$ DAC.

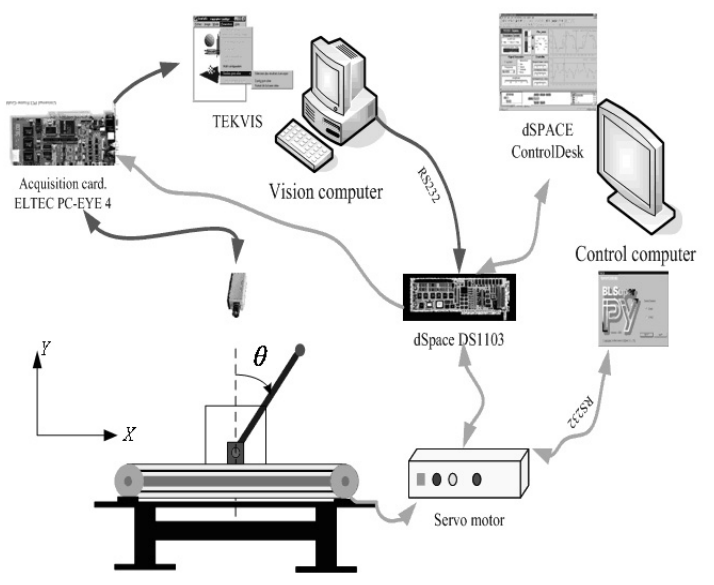

Figure 1. Vision based inverted pendulum setup 3) The actuator: This part is composed of a AC servo motor (SANYO DENKI PY2A015A3-A3). It's driven by a dSpace computer input/output card via the power amplifier supplied with $240 \mathrm{~V}$. The AC motor delivers a nominal couple of $3.0 \mathrm{Nm}$ with a power of $200 \mathrm{~W}$. The platform returns the cart's continuous position through a $8 \mu \mathrm{m}$ resolution incremental encoders equipped with the $\mathrm{AC}$ motor.

4) The vision system: Instead of using hi-tech digital cameras capable of higher sampling rates (up to 2000 frames/sec), higher spatial resolution (up to $4000 \times 4000$ pixels) and improved signal/noise ratio than their analog counterparts, we used a low cost IR CCD (Jai M50 IR) camera with a sampling rate of 25 frames/sec and a low resolution of $640 \times 480$ pixels in non-interlaced mode. It is linked to a vision computer which constitutes an image acquisition card ELTEC PC-EYE 4 and an image processing software TEKVIS. 


\section{Visual Measurement}

The presented vision system is used to determine the pendulum's upper tip $\left(x_{w}, y_{w}\right)$ image coordinates (in pixels) and transmits them to the control computer via the RS-232 serial communication port. In order to synchronize the camera with the control algorithm, the camera is triggered by an external periodical pulse signal, generated via the dSpace card with a sampling period equal the acquisition-processing-transfer time. Measurements made from image frames are noisy in nature due to changes in the environment's illumination, camera's slightly oblique position, and the deformations caused by the camera's wide angle lens. As soon as the control computer receives the pendulum's image coordinates in pixels, a four step TSAI calibration method [12] is carried out in the control system to acquire the pendulum's upper tip's real position $\left(x_{C}, y_{C}\right)$.

\subsection{Four step TSAI Calibration Method}

Step 1. Transformation from the calibration coordinate frame $\bar{p}_{w}=\left[x_{w}, y_{w}, z_{w}\right]$ to the camera coordinate frame

$\bar{p}_{s}=\bar{R}_{c} \bar{p}_{w}+\bar{t}_{c}$

where $\bar{p}_{s}=\left[x_{s}, y_{s}, z_{s}\right]$ is the point coordinates in camera coordinates, $R_{c}$ and $t_{c}$ are the rotation (orthonormal $3 \times 3$ matrix) and translation $(3 \times 1$ vector $)$ between the calibration frame and camera frames.

Step 2. Ideal perspective projection $\left(x_{p}, y_{p}\right)$ of $\left(x_{s}, y_{s}, z_{s}\right)$ to the image sensor

$x_{p}=f \frac{x_{s}}{z_{s}}, y_{p}=f \frac{y_{s}}{z_{s}}$

where $f$ is the focal length of the camera.

Step 3. Lens distortion moves the actual location of the projection to

$$
\begin{aligned}
x_{p}^{\prime} & =x_{p}\left(1+K_{1} r^{2}+K_{2} r^{4}\right) \\
& +2 P_{1} x_{p} y_{p}+P_{2}\left(r^{2}+2 x_{p}{ }^{2}\right) \\
y^{\prime} & =y_{p}\left(1+K_{1} r^{2}+K_{2} r^{4}\right) \\
& +P_{1}\left(r^{2}+2 x_{p}{ }^{2}\right)+2 P_{2} x_{p} y_{p}
\end{aligned}
$$

where $r=\sqrt{x_{p}{ }^{2}+y_{p}{ }^{2}}, K_{1}$ and $K_{2}$ are the coefficients of radial distortion, $P_{1}$ and $P_{2}$ are the coefficients of tangential distortion.

Step 4. Transformation to image coordinates
$x_{C}=N_{x}\left(\alpha \cdot x_{p}{ }_{p}+c_{x}\right) / S_{x}$

$y_{C}=N_{y}\left(y_{p}^{\prime}+c_{y}\right) / S_{y}$

where $N_{x}$ and $N_{y}$ are the number of the effective image points in horizontal and vertical directions, $\alpha$ is the correction to the aspect ratio, $S_{x}$ and $S_{y}$ are the dimensions of the effective image sensor, $\left(c_{x}, \mathrm{c}_{y}\right)$ is the principal point that is identical to the center of radial distortion.

Now, using the difference between the measurements and the model the camera parameters can be solved by minimizing the total squared error

$e^{2}=\sum_{i=1}^{N}\left(\varepsilon_{x i}{ }^{2}+\varepsilon_{y i}{ }^{2}\right)$

Before each use, the operators need to carry out a calibration work with a vertical fixed inverted pendulum. Then, this one will move according to a grid. For each point of the grid, the vision system will acquire the pendulum's upper tip coordinates and compare it with the actual continuous value measured by encoders. With the presented method, the camera's intrinsic parameters $K_{l}$, $K_{2}, P_{1}, \quad P_{2}, \quad c_{x}, \quad c_{y}, \alpha, f$ and extrinsic parameters $R_{c}$ and $t_{c}$ can be calculated.

Figure 2 illustrates the referred TSAI calibration method to the vertical fixed pendulum system. Comparing to the continuous encoder measurements, the TSAI corrected results are very satisfactory to points with only the linear correction even in the border sides. It should be noted that the error is more important on the sides (about $5 \mathrm{~mm}$ ). However, this is not very important, because these zones correspond to an unstable pendulum angle which isn't used in the stabilization control (cf. section 5).

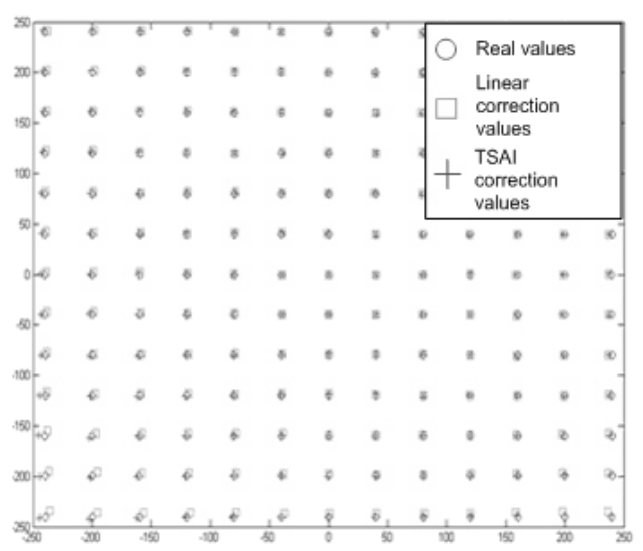

Figure 2. TSAI calibration results 


\subsection{Pendulum's Angle Computation}

According to the used vision system, the only accessible information is the coordinate $\left(x_{C}\right.$, $y_{C}$ ) of the pendulum's upper tip projection on the $x-y$ plane via the vision system and the cart's continuous position $x$. In these conditions, according to Figure 1, $\theta$ can be computed as follows

$\theta=\sin ^{-1}\left[\left(x_{C}-x\right) / 2 l\right]$

In our case, only sampled and delayed measurements of the camera are available

$\left(x_{C}\left(k t_{e}-T_{e}\right), y_{C}\left(k t_{e}-T_{e}\right)\right)=\left(x_{C, k-q}, y_{C, k-q}\right)$

With $t_{e}$ the camera's sampling cycle, and $T_{e}=q t_{e}$ where $k, q$ are integers, $T_{e}$ represents the delay time corresponding the time necessary for data acquisition, processing and transfer. In our vision system, $t_{e}=40 \mathrm{~ms}$ and $q=1$.

Finally, from (6) and (7), $\theta_{\mathrm{k}-1}$ is computed, the pendulum's angle feedback obtained in a sampled and delayed format.

\section{Modelling of the Cart-Inverted Pendulum System}

By referring to the methods proposed in [13], [14], the cart-inverted pendulum can be modeled as

$\ddot{x}=\left(-\dot{x}+k_{m} u\right) / \tau_{m}$

$\ddot{\theta}=-2 \zeta \omega_{n} \dot{\theta}+\omega_{n}^{2} \sin \theta-K \cos \theta \ddot{x}$

where $\omega_{n}=\sqrt{m g l /\left(J+m l^{2}\right)}$ is the natural frequency, $\quad \zeta=B_{r} /\left[2 \omega_{n}\left(J+m l^{2}\right)\right] \quad$ is the damping ratio, $K=m l /\left(J+m l^{2}\right)$ is the gain, and $\mathrm{u}, \mathrm{k}_{\mathrm{m}}$ and $\tau_{m}$ are the control input tension, the overall gain and the time constant of the cart-motor system. Here $\theta$ is the angle of the pendulum with y-axis, 1 is the length from the pendulum's center of gravity to the pivot, $m$ is the mass of the pendulum, $B_{r}$ is the viscous damping constant between the pendulum and the cart, $g$ is the gravitational acceleration, $\mathrm{J}=\left(\mathrm{ml}^{2}\right) / 3$ is the pendulum momentum of inertia and $\mathrm{x}$ is the cart's position on $\mathrm{x}$-axis.

The modelling cart-inverted pendulum system is a unstable nonminimum phase system, because under the assumptions $\mathrm{u}=0$ and $\mathrm{x}=0$, the system

$\ddot{\theta}+2 \zeta \omega_{n} \dot{\theta}-\omega_{n}^{2} \sin \theta=0$

has a positive eigenvalue. According to [15], input-output feedback linearization dose not pre-stabilize but only pre-compensates the system dynamics.

\section{Robust Hybrid Stabilization Control}

The main vision based hybrid control architecture, which is illustrated in Fig. 3, consists a Jumping-up control and a two loops cascade stabilization control. The commutation between the Jumping-up control and the two loops stabilization control is switched under a logic-based switch mechanism. The stabilization two cascade loops includes the inner loop (a linearization and the stabilization control of the pendulum based on PCROLO), and the outer loop (a Lyapunov based control for the unstable internal system having lower dynamics than that of the pendulum).

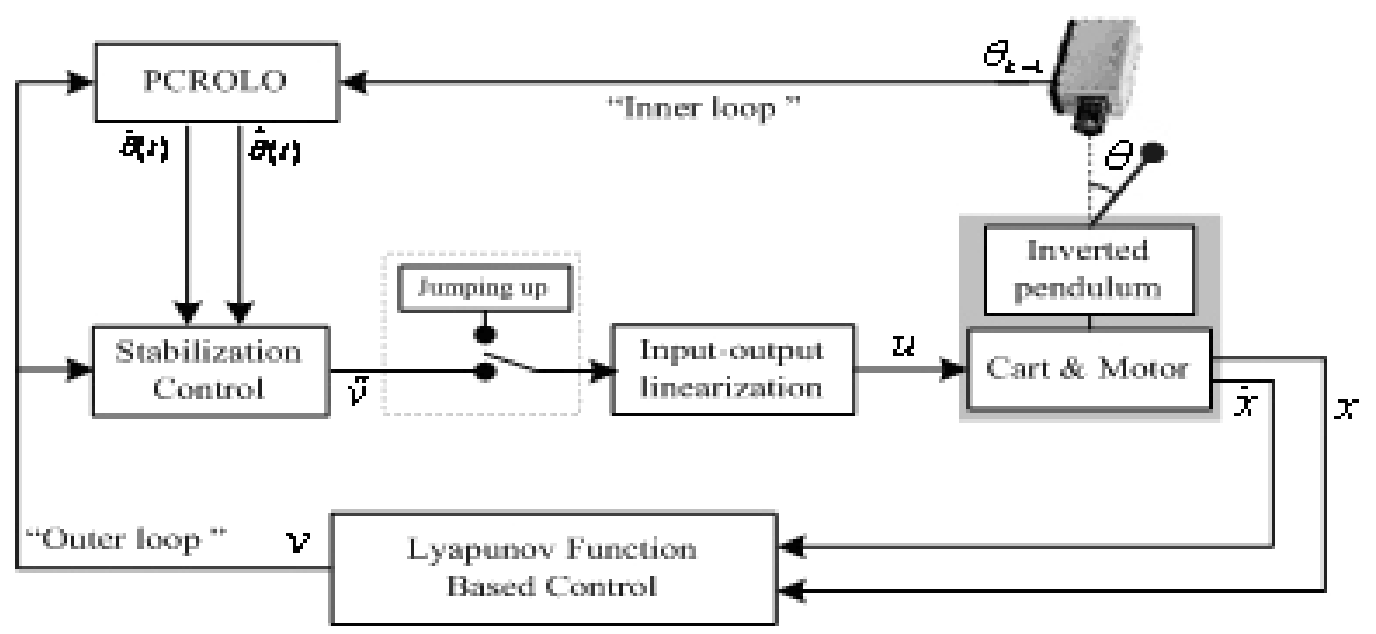

Figure 3. Hybrid control architecture for vision based cart-inverted pendulum 


\subsection{Jumping up control}

Normally, the proposed two loops cascade stabilization control method is valid for a big inclined initial pendulum angle $\left(\theta=50^{\circ}\right)$, but in the reason of the used motor's limited torque support in our real CIP system, we have to add a Jumping-up (Bang-Bang) control. The energy based swing-up control used in [16], [17], [18], [19] or like [20] a PD position controller to swing up the pendulum from the downward position to upward position can not be used here. This is because these controllers introduce an oscillation effect to move the pendulum to upright. However, in our systems, because of the circular shock absorber, the pendulum's angular position is limited above the horizontal plane.

The proposed Jumping-up control's main idea is simple. It comes from a basic everyday life example: considering a person leaned against a backward seat in a car, applying an appropriate accelerating $\left(t \in\left[0, t_{1}\right]\right)$, null $\left.\left.(t \in] t_{1}, t_{2}\right]\right)$ and decelerating $\left.\left.(t \in] t_{2}, T\right]\right)$ acceleration to the car, the person's body will incline forward. Inspired from this experience, the cart of CIP system is considered as the car and the pendulum as the person's body on the car. To facilitate the balancing CIP system, the ideal situation at the end of the Jumping-up control is that the cart's position $x(T)=0$ and speed $\dot{x}(T)=0$ under $x(0) \neq 0$. For a simplification sake, one notes $x_{0}=x(0), x_{T}=x(T)$ and $\varepsilon=\operatorname{sign}(x(0))$.

In (8) one introduces $\tilde{v}=\left(-\dot{x}+k_{m} u\right) / \tau_{m}$, which leads to

$\ddot{x}=\tilde{v}$.

First step: $t \in\left[0, t_{1}\right]$, with $x_{0} \neq 0$ and $\tilde{v}=-\varepsilon M, M>0$. By integration of $\ddot{x}=\tilde{v}$, one has

$x_{t_{1}}=x_{0}-\varepsilon M t_{1}^{2}$ and $\dot{x}_{t_{1}}=-\varepsilon M t_{1}$.

Second step: $\left.t \in] t_{1}, t_{2}\right]$, with $\tilde{v}=0$. One has

$x_{t_{2}}=x_{0}+\varepsilon M t_{1}^{2} / 2-\varepsilon M t_{1} t_{2}$ and $\dot{x}_{t_{2}}=\dot{x}_{t_{1}}$.

Third step: $\left.t \in] t_{2}, T\right]$, with $x_{T}=0, \tilde{v}=\varepsilon \alpha M$, $\alpha>0$. One has the relations of

$t_{1}=\alpha\left(T-t_{2}\right)$ and
$M=\varepsilon x_{0} /\left[T\left(t_{1}+\alpha t_{2}\right)-t_{1}^{2}+\alpha t_{2}^{2}+\alpha T^{2} / 2\right]>0$.

By adjusting the parameters $\left(x_{0}, T, \alpha, t_{2}\right)$ experimentally and considering the constraint on the position of the cart, the proposed strategy can jump up the pendulum to the unstable equilibrium zero under the pendulum's maximum angular position.

The switching is activated when the visual feedback shows that the pendulum is close to the upright position $\left(\left|\theta_{k-1}\right| \leq 0.2\right)$. This logicbased mechanism is realized by a RS trigger. The reason of choosing a big switch value for $\theta$ is to compensate the camera sensor's big time delay to produce a smoother switch under pendulum's small angular position $\theta$ and speed $\dot{\theta}$. This strategy has been tested successfully in the real vision based CIP system.

\subsection{Inner loop: PCROLO based stabilization control}

Under the assumption that the pendulum's angular position $\theta$ and velocity $\dot{\theta}$ are well estimated via PCROLO from $\theta_{\mathrm{k}-1}$, a stable inverted pendulum dynamics can be imposed by introducing a new control $\mathrm{v}$, a new gain $K_{2}$, a new natural frequency $\Omega_{n}$ and a new damping ratio $\mathrm{Z}$ defined as

$$
\begin{gathered}
-2 \zeta \omega_{n} \dot{\theta}+\omega_{n}^{2} \sin \theta-K \cos \theta \tilde{v}= \\
-2 Z \Omega_{n} \dot{\theta}-\Omega_{n}^{2} \theta+K_{2} v
\end{gathered}
$$

From (12), the following relation between $\tilde{v}$ and $\mathrm{v}$ is obtained:

$\tilde{v}=\frac{-2\left(\zeta \omega_{n}-Z \Omega_{n}\right) \dot{\theta}+\Omega_{n}^{2} \theta+\omega_{n}^{2} \sin \theta-K_{2} v}{K \cos \theta}$

with $|\theta|<\pi / 2$.

After the transformation, one gets

$\ddot{x}=\frac{-2\left(\zeta \omega_{n}-Z \Omega_{n}\right) \dot{\theta}+\Omega_{n}^{2} \theta+\omega_{n}^{2} \sin \theta-K_{2} v}{K \cos \theta}$

$\ddot{\theta}=-2 Z \Omega_{n} \dot{\theta}-\Omega_{n}^{2} \theta+K_{2} v$.

From (9) we obtain the linearized pendulum's state space model

$\dot{\Theta}(t)=A \Theta(t)+B v(t)$

with $A=\left[\begin{array}{cc}0 & 1 \\ -\Omega_{n}^{2} & -2 Z \Omega_{n}\end{array}\right], B=\left[\begin{array}{c}0 \\ K_{2}\end{array}\right]$ and $\Theta(t)=\left[\begin{array}{c}\theta(t) \\ \dot{\theta}(t)\end{array}\right]$. 
The problem is to estimate the continuous position $\theta(t)$ and velocity $\dot{\theta}(t)$ from $\theta_{\mathrm{k}-1}$. In order to solve this problem, we develop a specific PCROL observer. This observer combines a Reduced Order Discrete Luenberger Observer (RODLO) and two Piecewise Continuous Systems (PCS) as defined in [13].

A PCS $\Sigma\left(\left\{\mathrm{kt}_{\mathrm{e}}\right\}, \mathrm{A}, \mathrm{B}, \mathrm{C}\right)$ is characterized by a first continuous input $\varphi(t)$, a second input $\psi(\mathrm{t})$ sampled at discrete instants $\mathrm{kt}_{\mathrm{e}}$, three matrices $\mathrm{A}, \mathrm{B}, \mathrm{C}$, a state vector $\mathrm{x}(\mathrm{t})$ and an output vector $y(t)$. In these conditions, the functioning equations is defined as

$$
\begin{aligned}
& \begin{aligned}
& x_{k}^{0}= x_{k}(0)=\psi\left(k t_{e}\right), \quad \forall t \in\left\{k t_{e}\right\} \\
& x_{k}(t)=\exp \left(A\left(t-k t_{e}\right)\right) x_{k}^{0}+ \\
&+\int_{k t_{e}}^{t} \exp A(t-\tau) B \varphi(\tau) d \tau, \\
&\forall t \in] k t_{e},(k+1) t_{e}[
\end{aligned} \\
& y_{k}(t)=C x_{k}(t) . \quad \forall t \in[0,+\infty[
\end{aligned}
$$

In order to observe $\Theta(t)$, choose $A$ and $B$ as in (16) and $\mathrm{C}=\mathrm{I}_{2}$ According to Figure 4, the PCROLO is constructed as follows:

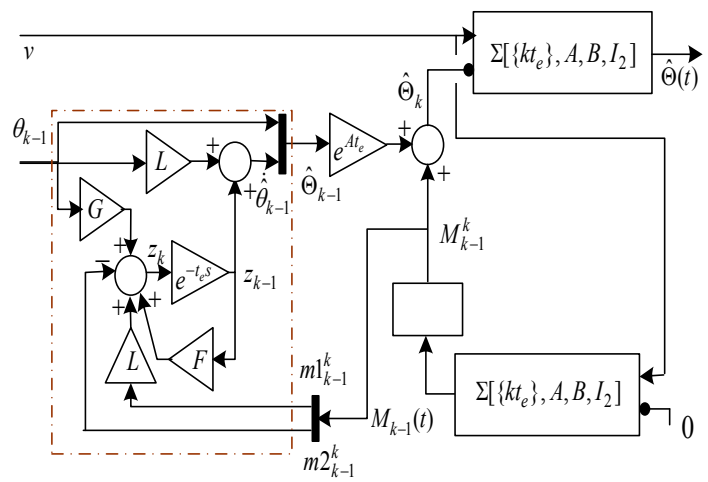

Figure 4. PCROLO observer

1) First step: PCS I. Using the PCS I with $\varphi(t)=v(t)$ and $\psi(t)=0$, one obtains

$M_{k-1}(t)=\int_{(k-1) t_{e}}^{t} \exp A(t-\tau) B v(\tau) d \tau$.

By sampling (Zero-Order-Holder) at each $\mathrm{kt}_{\mathrm{e}}$, one has

$$
\begin{aligned}
M_{k-1}^{k} & =\int_{(k-1) t_{e}}^{k t_{e}} \exp A\left(k t_{e}-\tau\right) B v(\tau) d \tau= \\
& =\left[\begin{array}{ll}
m 1_{k-1}^{k} & m 2_{k-1}^{k}
\end{array}\right]^{T} .
\end{aligned}
$$

2) Second step: RODLO. $\Theta_{k-1}$ is estimated by a RODLO defined as

$$
\begin{aligned}
& z_{k}=F z_{k-1}+G \theta_{k-1}+\left(m 2_{k-1}^{k}-L m 1_{k-1}^{k}\right), \\
& z_{k-1}=\dot{\hat{\theta}}_{k-1}-L \theta_{k-1}
\end{aligned}
$$

where $F, G$ and $L$ are defined from $\exp \left(A t_{e}\right)=\left[\begin{array}{ll}f_{11} & f_{12} \\ f_{21} & f_{n}\end{array}\right] \quad$ as: $\quad F=\left(f_{22}-L f_{12}\right)$, $G=\left(f_{22}-L f_{12}\right) L+\left(f_{21}-L f_{11}\right)$,

$L=\left(f_{22} / f_{12}\right) \in R \quad$ (maximizes RODLO's convergence speed).

Estimating $\dot{\theta}_{k-1}$ by $\dot{\hat{\theta}}_{k-1}=z_{k-1}+L \theta_{k-1}$, one gets $\hat{\Theta}_{k-1}$, then $\hat{\Theta}_{k}$, by integration of (16) on the time interval $\left[(k-1) t_{e}, k t_{e}[\right.$

$\hat{\Theta}_{k}=\exp \left(A t_{e}\right) \hat{\Theta}_{k-1}+M_{k-1}^{k}$.

3) Third step: PCS II. Using the PCS II, with $\varphi(\mathrm{t})=\mathrm{v}(\mathrm{t})$ and $\psi(t)=\hat{\Theta}_{k}$, one has

$$
\hat{\Theta}(t)=\exp (A t) \hat{\Theta}_{k}+\int_{k t_{e}}^{t} \exp A(t-\tau) B v(\tau) d \tau
$$

\subsection{Outer loop: Lyapunov function based control}

The main idea here is to introduce an angular reference $\theta_{\text {ref }}$ as an intermediate for the pendulum angular position in the aim of ensuring the stability of the vision based CIP system and coupling the control $\mathrm{u}$.

Referring to [15], [21], the quasi-steady state assumption in (8) and (9) is applied, the inverted pendulum system is brought to $\ddot{\theta}=\dot{\theta}=0$, and $\theta=\theta_{\text {ref. }}$ Thus the simplified dynamic system becomes

$$
\begin{aligned}
& \ddot{x}=\tilde{v}=\left[\omega_{n}^{2} \tan \left(\theta_{r e f}\right)\right] / K, \\
& v=\left(\Omega_{n}^{2} \theta_{r e f}\right) / K_{2} .
\end{aligned}
$$

Based on this internal simplified pendulum system, a Lyapunov candidate function is defined as follows

$$
V(x, \dot{x})=\left(\chi x^{2}+\delta \dot{x}^{2}\right) / 2 \text {, with } \chi, \delta \geq 0 .
$$

In order to ensure the Lyapunov derivation's negativity

$$
\dot{V}(x, \dot{x})=\dot{x}(\chi x+\delta \ddot{x})=\dot{x}(\chi x+\delta \tilde{v})<0,
$$

a particular function which stabilizes its nonminimum internal dynamics is defined as 


$$
\chi x+\delta \tilde{v}=-\mu\left[1-e^{-\left(\chi x^{2}+\delta \dot{x}^{2}\right) / 2}\right] \dot{x}, \quad \mu>0 .
$$

Therefore, the negativity of the chosen Lyapunov function is assured, and from (20), one gets the control

$$
\tilde{v}=-\left[\chi x+\mu\left(1-e^{-\left(\chi x^{2}+\delta \dot{x}^{2}\right) / 2}\right) \dot{x}\right] / \delta .
$$

Replacing (22) in (19), the intermediate value $\theta_{\text {ref }}$ is computed, and then by substituting $\theta_{\text {ref }}$ in (20), we have

$$
v=\tan ^{-1}\left\{-K\left[\chi x+\mu\left(1-e^{-\left(\chi x^{2}+\delta \dot{x}^{2}\right) / 2}\right) \dot{x}\right] /\left(\delta \omega_{n}{ }^{2}\right)\right\} \Omega_{n}^{2} / K_{2}
$$

Finally from (8), (22), u can be calculated as

$$
u=-\left\{\tau_{m} \chi x+\left[\tau_{m} \mu\left(1-e^{-\left(\chi x^{2}+\delta \dot{x}^{2}\right) / 2}\right)-\delta\right] \dot{x}\right\} / k_{m} \delta .
$$

\section{Simulation and Experimental Results}

In the experimental vision based system the mass of the cupreous pendulum is $560 \mathrm{~g}$ and its length is $32.4 \mathrm{~cm}$, the viscous friction between the pendulum and the cart is supposed small. Therefore, according to the modeling procedure, the inverted pendulum is characterized by $\zeta=0.0038$ and $\omega_{n}=6.739$. The cart-motor system is characterized with $k_{m}=2.92$ and $\tau_{m}=0.008$, and for the stabilization controller part we choose $K_{2}=\omega_{n}^{2}, \xi=1.2$, $\Omega_{n}=\omega_{n}, \chi=2.5, \delta=10$ and $\mu=8$.

\subsection{Simulation results}

Figure 5 illustrates the two loops stabilization control under an initial condition $\left(\mathrm{x}_{0}=0.5 \mathrm{~m}\right.$, $\left.\theta_{0}=\pi / 3 \mathrm{rad}, \dot{x}_{0}=0 \mathrm{~m} / \mathrm{s}, \dot{\theta}_{0}=0 \mathrm{rad} / \mathrm{s}\right)$. It is important to note that that the application of quasi-stationary assumption does not bring any restriction, no permanent oscillation appears, neither on the cart's displacement, nor on the pendulum. The results of simulation illustrate perfectly that the proposed two loops control ensures the stability of the total CIP system.

To facilitate the reading of the performances of the proposed observer, we make a zoom of the figures 5b (pendulum's angular position) and $5 \mathrm{~d}$ (pendulum's angular speed) on the time interval $[0.2 \mathrm{~s}, 2.5 \mathrm{~s}]$, and theirs corresponding results are illustrated in figures $5 \mathrm{c}$ and $5 \mathrm{e}$. The proposed observer estimates very well the pendulum's continuous angular position and its angular speed by the delayed and sampled vision system's measurements $\theta_{k-1}$.

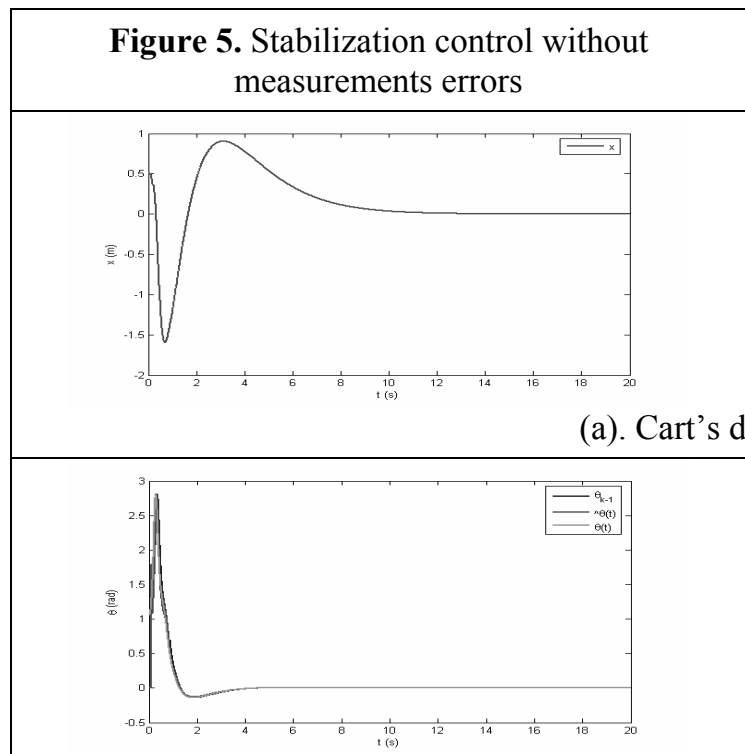

Figure 6. Stabilization control under measurements and identification errors

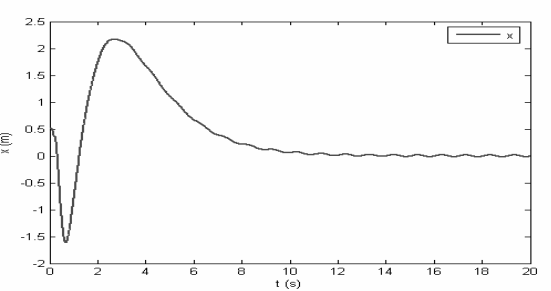

(a). Cart's displacement

(b). Pendulum's angular positions
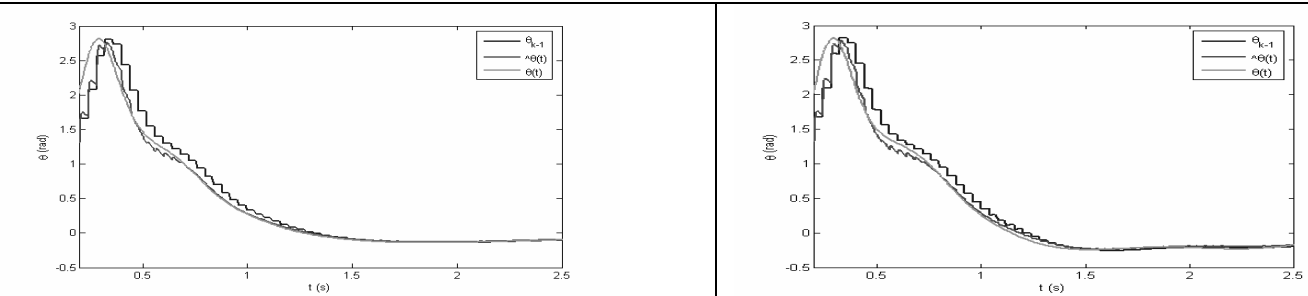

(c). Zoom of (b) in interval of [0.2 2.5] 


\begin{tabular}{|c|c|}
\hline $\begin{array}{l}\text { Figure 5. Stabilization control without } \\
\text { measurements errors }\end{array}$ & $\begin{array}{l}\text { Figure 6. Stabilization control under } \\
\text { measurements and identification errors }\end{array}$ \\
\hline 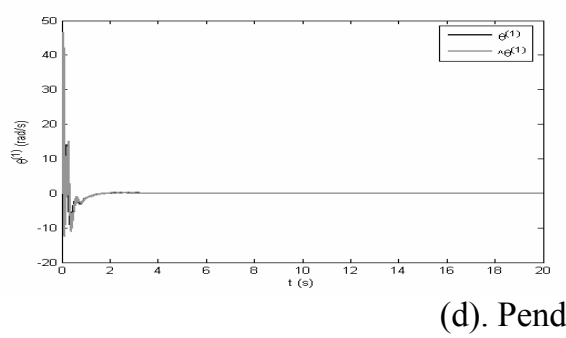 & 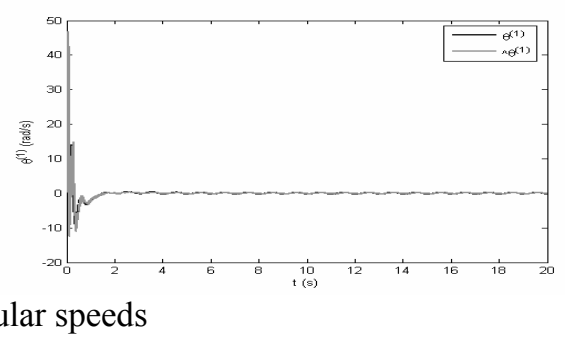 \\
\hline 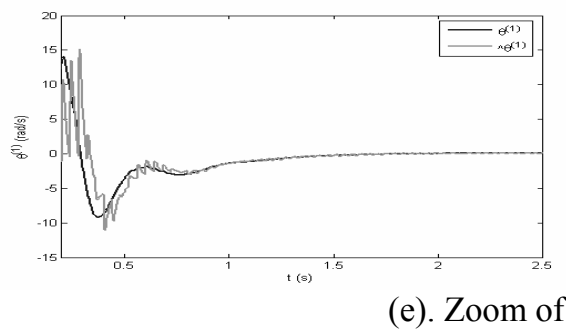 & 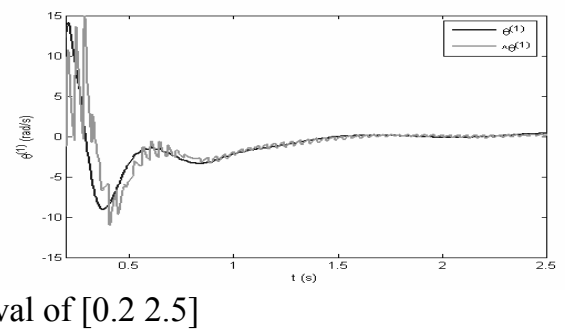 \\
\hline 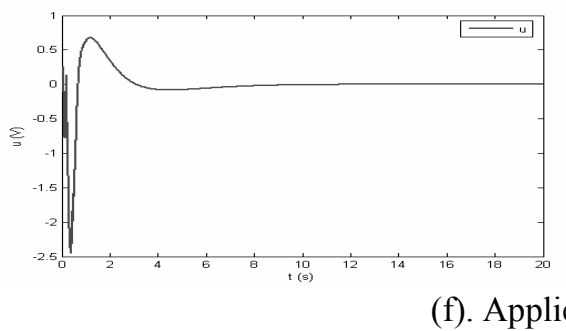 & ion control \\
\hline
\end{tabular}

As in all mechanical systems, the uncertain identification and the variation of the parameters (especially the coefficient of friction $\mathrm{B}_{\mathrm{r}}$ ) in function of $\theta(t)$ and $\dot{\theta}(t)$, are inevitable.

In order to study the robustness of the proposed method, we add simultaneously a parameter variation of $10 B_{r} \sin 2 \pi t$ in the parameter $B_{r}$ and a measurement error $0.02 \sin 2 \pi t$ on $\theta_{k-1}$. The corresponding simulation results are shown in Figure 6

It appears that, even in this case, the entire spherical pendulum system remains stable. The PCO still gives satisfactory estimations.

\subsection{Experimental results}

For the Jumping-up controller we select $\alpha=1, \quad T=0.14 \mathrm{~s}, \quad t_{1}=t_{2}=0.07 \mathrm{~s} \quad$ and $x_{0}=0.05 \mathrm{~cm}$, and for the stabilization controller part we choose $K_{2}=\omega_{n}^{2}, \xi=1.2$, $\Omega_{n}=\omega_{n}, \chi=100, \delta=10$ and $\mu=4$.

Note that the initial pendulum angular position $\theta_{0}=0.873 \mathrm{rd}=50^{\circ}$. So, we test the proposed control method on the real vision based CIP system under an initial condition $(x, \dot{x}, \theta, \dot{\theta})_{0}=(0.05,0,0.873,0)$.

The corresponding experimental results are given in Figures 7 and 8 which illustrate the Jumping-up and stabilisation control results and the stabilisation control results under manual perturbation. In both cases, the cart's position $\mathrm{x}$, the pendulum's angular positions $\theta_{k-1}$ and $\hat{\theta}(t)$, and the control $u_{x}$ are illustrated.

Fig. 7a shows that there remain residual oscillations limited to $\pm 3^{\circ}$ for the pendulum's angular position and $\pm 2 \mathrm{~cm}$ for the cart's position displacement. These oscillations are probably due to inaccurate identification of the viscous friction between the cart and the pendulum. Tests demonstrated that the cartpendulum system remains stable more than 10 hours (we did not test it beyond).

Fig. 8 illustrates the performances of the stabilization control under a manual perturbation. This perturbation which consists in trying to unbalance the entire pendulum system is applied at the pendulum's upper tip. It starts from about $4 \mathrm{~s}$ and ceases around $9 \mathrm{~s}$. One notices that the cart, under the perturbation, moves so as to ensure the balancing of the entire pendulum system. 


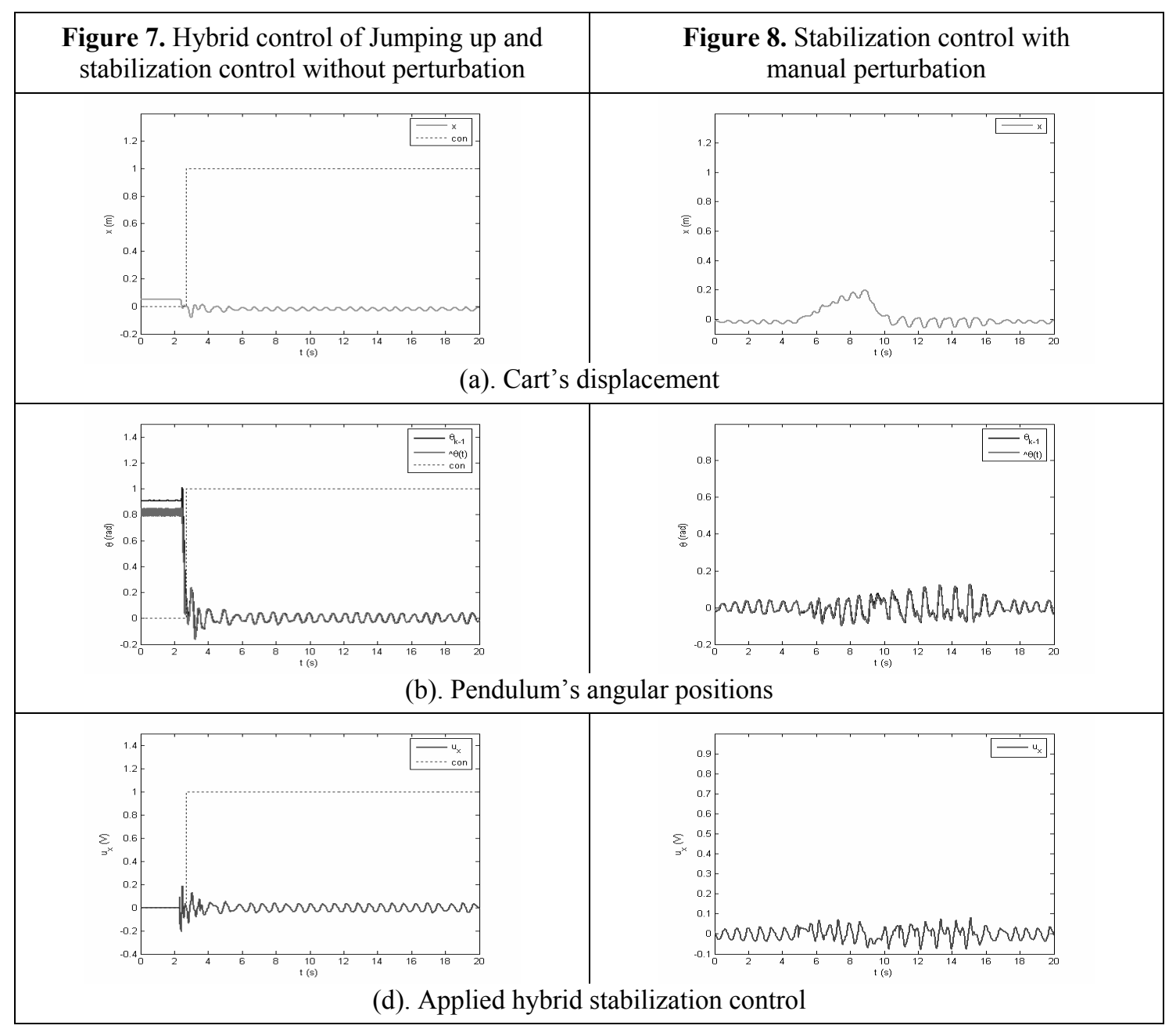

Moreover as soon as the perturbation ceases, the proposed control maintains the balance of the pendulum while bringing back the cart to its origin.

The illustrative videos of these experimentations are available on http://wwwlagis.univ-lille1.fr/ wang/Research_eng.html.

In spite of small oscillations, the experimental tests demonstrate that the proposed method is able to maintain the pendulum angular position upright, and keeping the cart's position around zero for $t \in[t,+\infty[$.

\section{Conclusions}

This article presents a direct and efficient method for controlling a vision based CIP system with a big time delay and a big inclined initial angular position. This is an underactuated mechanical system with nonlinear and nonminimum phase dynamics. The proposed control scheme is implemented on the dSpace Digital Signal Processing card via ControlDesk integrated with Matlab / Simulink platform. The experimental results demonstrate the effectiveness and robustness of the proposed approach.

\section{REFERENCES}

1. HUTCHINSON, S., G. D. HAGER, P. I. CORKE, A Tutorial on Visual Servo Control, IEEE Trans. on Robotics and Automation, Vol. 12(5), 1996, pp. 651-670.

2. ARMANDO, A. R., P. M. RICHARD, C. OGUZHAN, D. THANATE, Description of a Modelling, Simulation, Animation, and Real-tile Control (MoSART) Environment for a Class of Electromechanical Systems, IEEE Trans. on Education, Vol. 48(3), 2005, pp. 359-374.

3. SANCHEZ, J., S. DORMIDO, R. PASTOR, F. MORILLA, A Java / Matlab-based Environment for Remote Control System Laboratories: 
Illustrated with an Inverted Pendulum, IEEE Trans. on Education, Vol. 47(3), 2004, pp. 321-329.

4. ESPINOZA-QUESADA, E. S., L. E. RAMOS-VELASCO, Vision Based control of an Underactuated System Using a Reduced Observer, Proc. of Electronics, Robotics and Automotive Mechanics Conf., Vol. 1, Sept. 2006, pp. 9-14.

5. WENZEL, L., N. VAZQUEZ, D. NAIR, R. JAMAL, Computer Vision Based Inverted Pendulum, Proc. 17th IEEE Instrumentation and Measurement Tech. Conf.. Vol. 3, pp.1319-1323, 2000.

6. MAGANA, M. E., F. HOLZAPFEL, Fuzzy-logic Control of an Inverted Pendulum with Vision Feedback, IEEE Trans. on Education, Vol. 41(2), 1998, pp. 165-170.

7. FUKUDA, K. I., S. USHIDA, K. DEGUCHI, Just-in-time Control Image-based Inverted Pendulums with a Time-delay, Proc. SICE-ICASE International Joint Conf., Busan, Korea, October 2006, pp. 4016-4021.

8. KONCAR, V., C. VASSEUR, Control of Linear Systems Using Piecewise Continuous Systems, IEE Control Theory \& Applications, Vol. 150(6), 2003, pp. 565-576.

9. CHAMROO, A., I. SIMEONOV, C. VASSEUR, N. CHRISTOV, On the Piecewise Continuous Control of Methane Fermentation Processes, Studies in Informatics and Control, Vol. 17(2), 2008, pp. 181-188.

10. WANG, H. P., C. VASSEUR, A. CHAMROO, V. KONCAR, Sampled Tracking for Delayed Systems Using Piecewise Functioning Controller, Proc. 4th IEEE Conf. on Computational Engineering in Systems Applications, Beijing, Oct. 2006, Vol. 2, pp. 1326-1333.

11. WANG, H. P., C. VASSEUR, A. CHAMROO, V. KONCAR, Hybrid Control for Vision Based Cart-inverted Pendulum System, Proc. of the American Control Conf., Seattle, June 2008.

12. HEIKKILA, J., O. SILVEN, A Fourstep Camera Calibration Procedure with Implicit Image Correction, Proc. of IEEE Computer Society Conf. on Computer Vision and Pattern Recognition, June 1997, pp.1106-1112.

13. CHEN, L., R. SMITH, Closed-loop Model Validation for an Inverted Pendulum Experiment via a Linear Matrix Inequality Approach, Proc. of the 36th CDC, San Diego, Dec. 1997, pp. 2565-2566.

14. DELEY, D. W., Controlling an Inverted Pendulum: Example of a Digital Feedback Control System, available: http://members.cox.net/srice1/pendulum/i ndex.htm, January 2007.

15. GUEMGHAR, K., B. SRINIVASAN, PH. MULLHAUPT, D. BONVIN, Analysis of Cascade Structure with Predictive Control and Feedback Linearization, IEE Control Theory \& Applications, Vol. 152(3), 2005, pp. 317-324.

16. ASTROM, K. J., K. FURUTA, Swinging Up a Pendulum by Energy Control, Automatica, Vol. 36, 2000, pp. 287-295.

17. BUGEJA, M., Non-linear Swing-up and Stabilizing Control of an Inverted Pendulum System, Proc. of. EUROCON, Ljubljana, 2003.

18. CHATTERJEE, D., A. PATRA, H. JOGLEKAR, Swing-up and Stabilization of a Cart-pendulum System Under Restricted Cart Track Length, System and Control Letters, Vol. 47(4), 2002, pp. 355-364.

19. SPONG, M. W., Energy Based Control of a Class of Underactuated Mechanical Systems, Proc. 13th IFAC World Congress, Vol. F, July 1996, pp. 431-436.

20. NUNDRAKWANG, S., T. BENJANARASUTH, J. NGAMWIWIT, N. KOMINE, Hybrid Controller for Swinging Up Inverted Pendulum System, Proc. of the 5th International Conf. on Information, Communications and Signal, Dec. 2005, pp. 488-492.

21. ISIDORI, A., Nonlinear Control Systems, Springer, Third edition, 1994. 